\title{
Chromaticity Measurement Using A Continuous Head-Tail Kicking Technique *
}

\author{
C.Y. Tan ${ }^{\dagger}$, Fermilab, Batavia, IL 60510, USA \\ V.H. Ranjbar ${ }^{\ddagger}$, Tech-X, Boulder, CO 80303, USA
}

\begin{abstract}
In the classical head-tail chromaticity measurement technique, a single large kick is applied transversely to the beam. The resulting phase difference between the head and the tail is measured and the chromaticity extracted. In the continuous head-tail kicking technique, a very small transverse kick is applied to the beam and the asymptotic phase difference between the head and the tail is found to be a function of chromaticity. The advantage of this method is that since the tune tracker PLL already supplies the small transverse kicks, no extra modulation is required.
\end{abstract}

\section{INTRODUCTION}

Continuous chromaticity control up the ramp and through the squeeze will be vital for operating the Large Hadron Collider (LHC). It is especially important at the beginning of the ramp because of snapback where chromaticity swings are expected to drift at a rate of $0.33 \mathrm{~s}^{-1}$ even with pre-programmed chromaticity correction tables. This is to be compared to the tolerance required for nominal $7 \mathrm{TeV}$ head-on collisions operations which is \pm 1 unit.[1]

Several techniques have been proposed for measuring chromaticity continuously. In this paper, we will discuss the continuous head-tail kicking technique. Contrast this to the traditional head-tail method which kicks the beam once transversely and because of chromaticity, the head and the tail de-phase differently.[2] When the phase difference between the head and the tail is measured, it can be shown that when the phases are maximally different, this difference is linearly related to the chromaticity. In contrast, the continuous head-tail kicking technique kicks the beam transversely at a frequency that is close to the betatron tune and the resultant constant phase difference between the head the tail is related to the chromaticity. The advantage of this method is that

- There is already a continuous transverse kick from the tune tracker PLL. It has already been demonstrated at Fermilab, RHIC, and SPS that these small kicks $\ll 1 \mu$ m do not blow up the emittance or cause beam lifetime problems.

- No extra modulations are required for the chromaticity measurement. For example, the traditional method

\footnotetext{
* Work supported by Fermi Research Alliance, LLC under Contract No. DE-AC02-07CH11359 with the United States Department of Energy, and US-LARP.

$\dagger$ cytan@fnal.gov

$\ddagger$ ranjbar@txcorp.com
}

for measuring chromaticity requires changing the RF frequency to change the momentum of the beam.

- The method is compatible with the tune tracker PLL.

\section{THEORY}

We can write down the transverse equation of motion for a single particle as

$$
\frac{d^{2} X(s)}{d s^{2}}+\frac{\omega_{Q}^{2}}{c^{2}} X(s)=0
$$

Here $X$ is the transverse position of the particles, $s$ is the longitudinal coordinate and $\omega_{Q}$ is the betatron frequency and $c$ is the speed of light. However, if the particle resides in an RF-bucket, we must consider its longitudinal motion inside the bucket and so the equation of motion becomes

$$
\frac{d^{2} X(s, \delta, z)}{d s^{2}}+\frac{\omega_{Q}^{2}(\delta)}{c^{2}} X(s, \delta, z)=0
$$

Here $z$ defines the longitudinal position relative to the centre of the RF-bucket and $\delta$ is the relative momentum difference from the "on momentum" particle. If we expand the betatron frequency to first order in $\delta$ we obtain

$$
\omega_{Q}(\delta)=\omega_{0} Q+\xi \omega_{0} \delta
$$

where $\omega_{0}$ is the revolution frequency, $Q=\omega_{Q} / \omega_{0}$ is the betatron tune and $\xi$ is the chromaticity and approximate the longitudinal motion inside the RF-bucket with

$$
\begin{aligned}
\delta(s) & =-\frac{\omega_{s}}{\eta c} r \sin \left(\frac{\omega_{s} s}{c}+\phi\right) \\
z(s) & =r \cos \left(\frac{\omega_{s} s}{c}+\phi\right)
\end{aligned}
$$

where $\omega_{s}$ is the synchrotron frequency, $(r, \phi)$ is position of the particle at $s / c=0$ and $\eta$ is the slip factor, then we can write down a linear transformation which maps $(z(0), \delta(0)) \equiv\left(z_{0}, \delta_{0}\right)$ to $(z(s), \delta(s))$

$$
\left(\begin{array}{c}
z(s) \\
\delta(s)
\end{array}\right)=\left(\begin{array}{cc}
\cos \left(\frac{\omega_{s} s}{c}\right) & \frac{\eta c}{\omega_{s}} \sin \left(\frac{\omega_{s} s}{c}\right) \\
-\frac{\omega_{s}}{\eta c} \sin \left(\frac{\omega_{s} s}{c}\right) & \cos \left(\frac{\omega_{s} s}{c}\right)
\end{array}\right)\left(\begin{array}{c}
z_{0} \\
\delta_{0}
\end{array}\right)
$$

We can also think of the map given by Eq. (6) as a frame that is rotating at the synchrotron frequency. Thus in this frame, Eq. (1) becomes

$$
\begin{array}{r}
\frac{d^{2} x(s, \delta, z)}{d s^{2}}+\omega_{0}^{2}\left[Q+\xi\left(\delta_{0} \cos \left(\omega_{s} s / c\right)\right.\right. \\
\left.\left.-\omega_{s} \tau_{0} \sin \left(\omega_{s} s / c\right)\right) / \eta\right]^{2} x(s, \delta, z)=0
\end{array}
$$


where $\tau_{0}=z_{0} / c$ and we have mapped $X \rightarrow x$ to remind us that we are in the rotating frame. Next, let us change variables to use turns $n$ rather than $s$, i.e.

$$
n=\frac{s}{2 \pi R} \quad \Rightarrow \quad \frac{d n}{d s}=\frac{1}{2 \pi R}
$$

where $R$ is the radius of the accelerator. In this variable, Eq. (7) with a weak sinusoidal kick becomes

$$
\begin{aligned}
& \frac{d^{2} x}{d n^{2}}+[2 \pi Q+ \\
& \left.2 \pi \xi\left(\delta_{0} \cos \left(2 \pi Q_{s} n\right)-\frac{\omega_{s} \tau_{0}}{\eta} \sin \left(2 \pi Q_{s} n\right)\right)\right]^{2} x \\
& \quad=\epsilon \lambda \cos 2 \pi Q_{k} n
\end{aligned}
$$

where $s / c=2 \pi n / \omega_{0}, Q_{s}=\omega_{s} / \omega_{0}$ is the synchrotron tune, $\epsilon \ll 1, Q_{k}$ is the frequency of the kick in tune units and $\lambda=1$ has the same dimensions as $x$ to keep the dimensions of the lhs and rhs of the o.d.e. correct. It is clear that Eq. (7) is Hill's equation with an external periodic forcing. A very good approximate solution in terms of trigonometric functions can be found by the Averaging Method.[3]

After obtaining the approximate solution for a single particle, we transform back to the laboratory frame and integrate over the particle distributions longitudinally and transversely. If we assume that the longitudinal distribution is stationary, i.e. "matched to the bucket", and the transverse distribution has zero emittance, we find that the phase difference $\Delta \psi$ of the head at $+\tau_{B}$ w.r.t. tail at $-\tau_{B}$ to be

$$
\Delta \psi\left(\xi, \tau_{B}\right)=+\frac{2 \xi \omega_{0} \tau_{B}}{\eta}
$$

\section{EXPERIMENT}

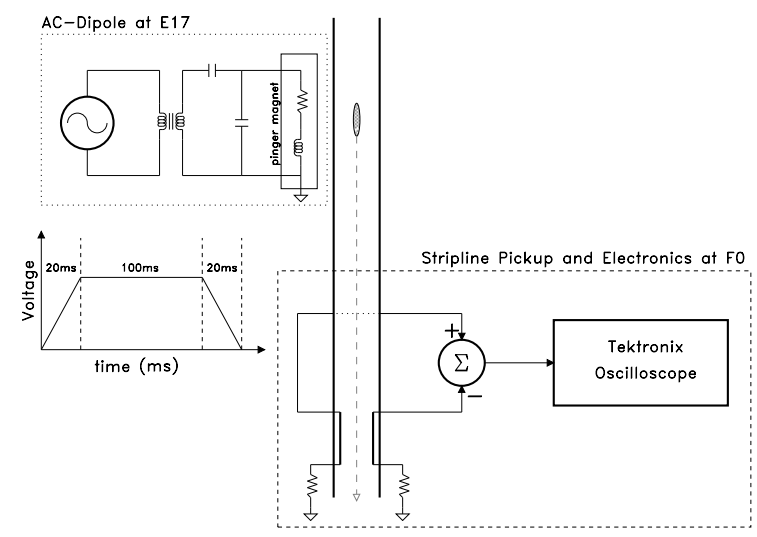

Figure 1: The experimental setup consists of an AC-dipole at E17 and a stripline with its electronics at F0. The ACdipole is ramped up to its set voltage in $20 \mathrm{~ms}$ and stays at flattop for about $100 \mathrm{~ms}$ (equivalent to about 10 synchrotron periods or 5000 turns) and then ramped down again in $20 \mathrm{~ms}$.

In the experiment, we inject one coalesced bunch, which contains about $330 \times 10^{9}$ protons, to be used for the entire experiment. We set the vertical chromaticity and then we kick the bunch transversely with the AC-dipole for a short period of time for each head tail phase measurement. The reason for turning off the AC-dipole is to keep the vertical emittance growth to a minimum. The frequency of the kick is set to $0.6 \times 10^{-3}$ tune units $\left(<Q_{s}=1.7 \times 10^{-3}\right)$ below the vertical betatron tune which is at 0.5776 . The transverse position of the head and the tail are measured with a fast sampling oscilloscope and the phase difference is calculated offline. After each change of chromaticity, we move the vertical tune if necessary to ensure that the betatron tune remains at 0.5776 . Despite having set the AC-dipole kick to its minimum value, the vertical emittance measured with the flying wires system, grows from $17 \pi \mathrm{mm} \cdot \mathrm{mrad}$ to $36 \pi \mathrm{mm} \cdot \mathrm{mrad}$ during the experiment. The sigma bunch length $\sigma_{B}$ did not change during this time and is $2.9 \mathrm{~ns}$.
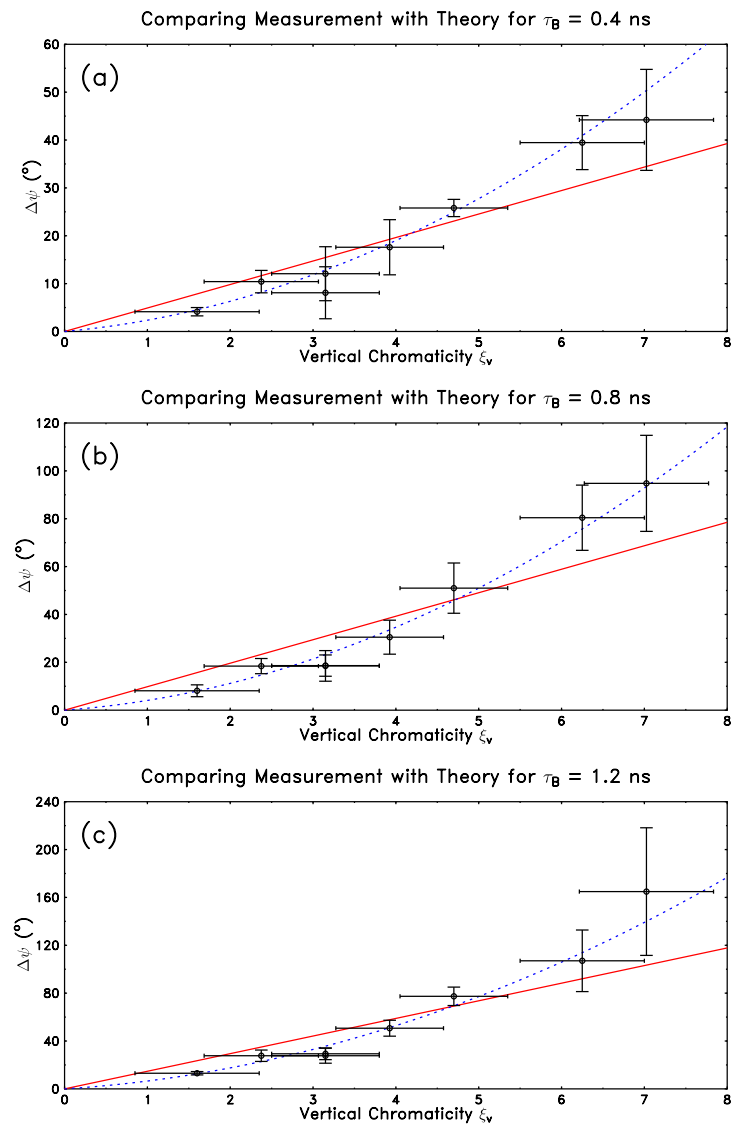

Figure 2: These plots show the data analysed for $\tau_{B}=$ $0.4 \mathrm{~ns}, 0.8 \mathrm{~ns}$ and $1.2 \mathrm{~ns}$. The red line is calculated from Eq. (10) and the blue dotted line is a quadratic fit.

\section{Results}

The measured phase difference $\Delta \psi$ for three values of $\tau_{B}=0.4 \mathrm{~ns}, 0.8 \mathrm{~ns}$ and $1.2 \mathrm{~ns}$ versus $\xi_{v}$ are shown in Figure 2. The red line is the expected $\Delta \psi$ as a function of $\xi_{v}$ from Eq. (10) where we have used the values $\omega_{0}=$ 
$2 \pi \times\left(47.712 \times 10^{3}\right) \mathrm{s}^{-1}$ and $\eta=0.0028$. It is obvious that the theory does not fit the data well. We suspect that there is a quadratic component $\xi_{v}^{2}$ that is not taken care of in the theory. By fitting the data with

$$
\Delta \psi=\xi_{v}\left(a \xi_{v}+b\right)
$$

where $a$ and $b$ are fit parameters, we can explore how good this hypothesis is. The blue curves in Figure 2 show the quadratic fit. It is clear from the plots that the quadratic fits are rather good

\section{Sources of the Quadratic Term}

There are several possible sources for the quadratic term $\xi_{v}^{2}$. Eq. (10) is derived by assuming that the RF bucket is linear and that the transverse emittance is zero. Another possibility is that the transverse impedance is influencing the phase shift between the head and the tail. Whether these possibilities are the sources for the quadratic term will have to be resolved with computer simulations.

We do not think that second order chromaticity is the source of the quadratic term because they tend to cancel out when we take the difference between the head and the tail. The best candidate for the source of the quadratic term would seem to come from the impedance in the beam pipe. A more careful analysis of the effect of transverse impedance is provided using a multi-particle simulation driven by a single kick, which includes linear chromaticity and the effect of a short range resistive wall wake field. In Figure 3 we can see that the maximum phase difference as a function of chromaticity acquires a quadratic term as the strength of the wake field is increased.

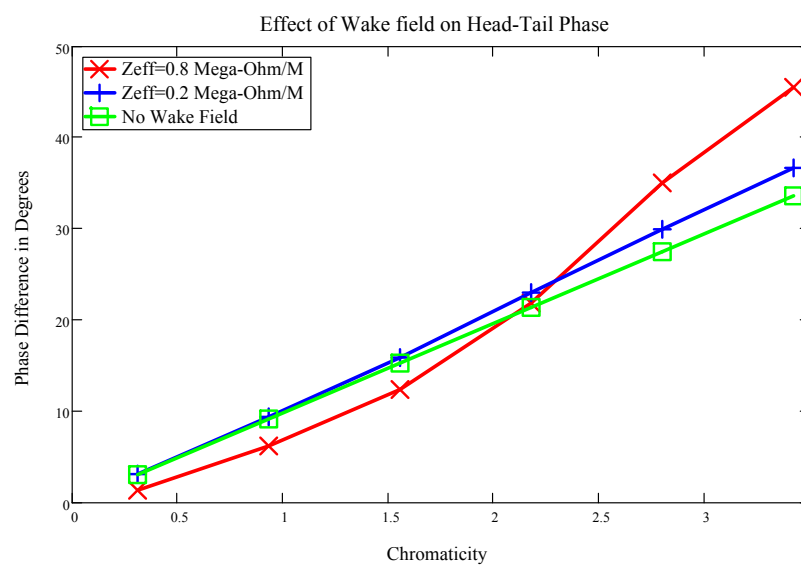

Figure 3: The effect of resistive wall wake field on the measured phase difference between the head slice $(+0.4 \mathrm{~ns}$ from bucket center) and the tail slice $(-0.4 \mathrm{~ns}$ from bucket centre) for a single kick. The red trace shows the effect of impedance at $0.8 \mathrm{M} \Omega / \mathrm{m}$, blue trace at $0.2 \mathrm{M} \Omega / \mathrm{m}$ and the green trace is for zero impedance.

\section{CONCLUSION}

We have used an analytic approximation for the weakly forced Hill's equation which describes the transverse motion of the beam in the presence of chromaticity for a single particle to derive a formula for the phase difference between the head and tail for a zero transverse emittance but non-zero longitudinal length bunch. From the measured data, we have found that there there is a quadratic term which is not taken care of by the theory. Several possible sources, with short range wake fields being the best candidate, of this term have been proposed which we will have to resolve with computer simulations.

\section{REFERENCES}

[1] S.Fartoukh \& J.P. Koutchouk, "On the Measurement of the Tunes, Coupling \& Detunings with Momentum and Amplitude in LHC", LHC-B-ES-0004 rev 2.0.

[2] D. Cocq et al, "The Measurement of Chromaticity Via a Head-Tail Phase Shift", http://www.slac.stanford.edu/pubs/ confproc/biw98/schmickler.pdf, 1998.

[3] C.Y. Tan \& V. Ranjbar, "Continuous Transverse Excitation for Measuring Chromaticity from the Head-Tail Phase Difference: An Analytic Approximation with Experimental Data", TM-2376, Fermilab, 2007. 